\title{
Processos de acreditação para o enfermeiro: um recorte histórico
}

\author{
Accreditation processes for nurses: a historical briefing
}

Procesos de acreditación para el enfermero: un retazo histórico

\section{Rosana Chami Gentill, Maria Cristina Sannal \\ 'Universidade Federal de São Paulo. Departamento de Enfermagem, Grupo de Estudos e Pesquisas em Administração e Gerenciamento em Saúde e Enfermagem. São Paulo, SP}

Submissão: $19 / 10 / 2008$

Aprovação: 05/01/2009

\section{RESUMO}

Para identificar, descrever e analisar os marcos históricos da acreditação de enfermeiros na área de cuidados críticos foi realizada pesquisa no LILACS e MEDLINE, identificando-se 16 publicações Que resultaram em 4 categorias. Nos Estados Unidos, o CCNE e a NLNAC se dedicam a avaliar cursos e instituições de ensino de Enfermagem, os enfermeiros generalistas são avaliados pelo NCLEX e os especialistas por entidades de classe. Também há interesse na acreditação de enfermeiros na Espanha, Canadá, África do Sul e Austrália. Nos EUA, o processo começou na década de 1940 e, na Espanha, na de 1990. Concluiu-se Que a certificação e acreditação profissional na Enfermagem são praticadas principalmente em países desenvolvidos e iniciaram-se no meio do século passado.

Descritores: Acreditação; História da enfermagem; Prática profissional; Educação continuada.

\begin{abstract}
To identify, describe and analyze the historical milestones of nurses' accreditation in the critical care area, a research was carried out at LILACS and MEDLINE, unearthing 16 publications, led to the 4 categories. In the USA, the CCNE and NLNAC focus on evaluating Nursing courses and educational institutions, whilst generalist nurses are evaluated by NCLEX, and experts are evaluated by professional associations. Accrediting nurses is also a matter of interest in Spain, Canada, South Africa and Australia. In the USA, the process began in the 40's, whilst in Spain it began in the 90's. It was concluded that the professional certification and accreditation in Nursing occur mainly in developed countries and began in the middle of the last century.

Descriptors: Accreditation; History of nursing; Professional practice; Education, continuing.
\end{abstract}

\section{RESUMEN}

Para identificar, describir y analizar los marcos históricos de la acreditación de enfermeros en el área de cuidados críticos, se llevó a cabo una investigación en el LILACS y MEDLINE que redundó en la identificación de 16 publicaciones, arrojaron 4 categorías. En los EE.UU., el CCNE y la NLNAC se dedican a la evaluación de cursos e instituciones de enseñanza de Enfermería, los enfermeros generalistas son evaluados por el NCLEX, mientras los especialistas son evaluados por las asociaciones profesionales. Igual hay interés en la acreditación de enfermeros en España, Canadá, Sudáfrica y Australia. En los EE.UU., el proceso se puso en marcha en los años 1940, mientras en España se dio en los años 1990. Se concluyó que la certificación y acreditación profesional en Enfermería se practican principalmente en países desarrollados y Que empezaron a mediados del siglo pasado.

Descriptores: Acreditación; História de la enfermería; Práctica profesional; Educación continua. 


\section{INTRODUÇÃO}

Há um crescente interesse sobre a temática certificação e acreditação, Que se evidencia pela preocupação com a elevação no contingente de formação de enfermeiros, e pelo fato desta ser uma profissão em constante transformação, apontando para a necessidade da criação de um modelo de acreditação para este profissional.

Nessa perspectiva, o interesse e a preocupação dos enfermeiros paulistas com a Qualidade do profissional já resultou em proposição de modelo de acreditação Que, no entanto, não chegou a ser implementado( ${ }^{(1)}$.

A dificuldade em conceituar acreditação em Enfermagem é uma medida do Quanto esse assunto ainda é pouco explorado na literatura científica. O Consórcio Brasileiro de Acreditação (CBA) ${ }^{(2)}$ define a acreditação como um processo de avaliação objetiva, baseada em padrões explícitos, Que visa estimular a melhoria contínua e sustentada dos cuidados oferecidos. Caracteriza-se por ser uma avaliação externa, de caráter voluntário, periódico e reservado. Esse conceito, no entanto, é voltado para a avaliação de instituições e não de profissionais.

Por outro lado, de acordo com a Associação Brasileira de Norma Técnicas $(\mathrm{ABNT})^{(3)}$, certificação é uma modalidade de avaliação de conformidade a padrões pré-estabelecidos, realizada por uma organização independente das partes diretamente envolvidas na relação comercial. Certificar um produto, serviço ou sistema significa comprovar, junto ao mercado e aos clientes, Que a organização possui um sistema de fabricação controlado, garantindo Que as atividades especificadas estão de acordo com as normas. Ainda Que se refira a produtos, certamente o conceito não se aplica ao Que resulta de processos de formação de recursos humanos.

O sistema de acreditação na Enfermagem vem de duas influências: uma originada de preocupação do profissional com o bem estar e segurança da saúde pública, e outra como resposta à demanda do consumidor pela segurança e habilidade do enfermeiro para desenvolver tarefas altamente especializadas utilizando julgamento independente $e^{(4)}$.

A certificação para o enfermeiro nos EUA iniciou-se em 1940. Desde essa época, o número de certificações tem aumentado consideravelmente. O National Council's of Learning Extension (NCLEX) é a principal entrada para o exame de enfermeiros, considerando, para a classificação, a avaliação do conhecimento do enfermeiro em uma área específica de enfermagem ${ }^{(5)}$.

No Brasil, o Conselho Federal de Medicina $(\mathrm{CFM})^{(6)}$, com a Resolução CFM no 1.772/2005, instituiu o Certificado de Atualização Profissional (CAP) para os portadores dos títulos de especialistas e certificados de áreas de atuação. Para tanto, criou a Comissão Nacional de Acreditação (CNA), para elaborar normas e regulamentos para esse fim, além de coordenar a emissão desses certificados. A partir de janeiro de 2006, médicos Que possuem títulos de especialista ou certificado de área de atuação terão cinco anos para somar 100 pontos em atividades de educação continuada e obter o "Certificado de Atualização Profissional". A participação é obrigatória para os Que se tornarem especialistas a partir do ano citado. As Sociedades de Especialista devem proporcionar, aos médicos, um mínimo de 40 créditos a cada ano, sendo 20 deles referentes a atividades de caráter regional ou estadual. Além disso, têm de disponibilizar um mínimo de 10 créditos anuais correspondentes a programas à distância (on-line ou impressos), o que equivale a 20 horas/aula.

As sociedades de especialistas têm papel importante nesta ação profissional, pois é delas Que partem iniciativas de caráter nacional, estadual ou regional, promovendo diferentes atividades de atualização para a obtenção dos créditos necessários para efeito de acreditação profissional.

Takahashi e Oguisso ${ }^{(7)}$, estudando o assunto, afirmaram Que esses programas estabelecem metas a serem alcançadas com a certificação do trabalhador, como "capacitar para o exercício das atividades profissionais e orientar a formação/força de trabalho com otimização dos recursos, pois informa e orienta a formação e as possibilidades de emprego, avaliando e acreditando as competências necessárias para esse exercício profissional e a empregabilidade, tanto na aprendizagem formal como na informal, além de constituir um instrumento de avaliação da formação".

Frente a essa multiplicidade de abordagens, e considerando oportuno oferecer embasamento teórico para aprofundar as discussões a respeito do tema, resolveu-se estudar o assunto para Que, conhecendo os padrões e critérios para a acreditação e certificação e os marcos históricos desses processos, também se possa subsidiar a criação de programas de avaliação de enfermeiros especialistas, particularmente na área de Enfermagem em Emergências.

\section{OBJETIVO}

Identificar, descrever e analisar os marcos históricos da acreditação profissional do enfermeiro em emergência, nos países Que a praticam.

\section{MÉTODO}

Estudo descritivo, de caráter histórico e de base bibliográfica, Que teve como população as produções científicas nacionais e internacionais relacionadas ao assunto, entre as Quais se encontrou o artigo mais antigo datado de 1975 e o mais atual de 2008.

A coleta de dados foi realizada nas bases de dados LILACS e MEDLINE, acessadas através da Biblioteca Virtual em Saúde ${ }^{(8)}$, empregando-se as expressões: "emergency nursing certification", "emergency nursing accreditation"e "accreditation nursing".

A coleta das indicações bibliográficas se deu diretamente das bases citadas, seguida da seleção de produções referentes ao assunto. Os textos foram selecionados em função do reconhecimento de sua vinculação ao assunto em estudo. Foram levados em consideração os Que continham informações sobre a definição do Que é certificação e acreditação, e informações sobre a história da certificação e acreditação na Enfermagem. Para tanto, foram lidos e analisados os resumos de todas as obras capturadas e excluídas aquelas Que continham informações de caráter diverso do assunto em estudo. Assim, de um total de 42 títulos, 26 foram excluídos por não abordarem a acreditação de enfermeiros.

Os 16 títulos selecionados foram capturados e depois foi realizada a leitura dos mesmos na íntegra, seguida de fichamento contendo: referência bibliográfica, síntese da produção e comentário pessoal da pesquisadora principal. 
As fichas foram então agrupadas por similaridade e pertinência temática e em ordem cronológica dos marcos históricos, a partir do Quê se construiu o esquema para elaboração do relato dos achados. As categorias de análise encontradas foram: "Conceituação sobre Acreditação e Certificação", "Acreditação de Cursos de Graduação em Enfermagem", "Acreditação dos Programas de Educação Continuada para Enfermeiros" e "Certificação e Recertificação de Enfermeiros Especialistas".

\section{RESULTADOS}

\section{Conceituação sobre Acreditação e Certificação}

Os conceitos de acreditação e certificação experimentam diferentes usos na literatura estudada. Barnum ${ }^{(9)}$, por exemplo, fez uma revisão sobre os três grandes caminhos para o enfermeiro obter certificação de competências: licença, certificação e acreditação. Para o autor, a enfermeira registrada (Registered Nurse - $R N$ ) ou a enfermeira com a licença para a prática (Licensed Practical Nurse - LPM) é portadora de uma licença individual e particular, decorrente de avaliação Que ateste ter adQuirido o conhecimento básico requerido para a prática segura.

A Licença é geralmente uma concessão do Estado e não do governo federal, dado Que os EUA são uma república federativa. O Council of State Boards of Nursing traça as diretrizes gerais para essa prática, tendo sido criado para favorecer a uniformidade nacional de métodos e a adoção de critérios harmônicos entre os Estados, embora tecnicamente com margem para Que cada um seja legalmente livre para definir os regulamentos para atribuição de licença e realização das provas.

A certificação para enfermeiros envolve uma prova individual para comprovar o nível alcançado pelo enfermeiro em alguma especialidade. Para obter essa licença o candidato tem Que contemplar um desempenho mínimo. Ela é orientada em direção a um espectro diferente do requerido para o generalista, comprovando Que as enfermeiras alcançaram, com êxito, uma competência de especialista. O processo de certificação é voluntário e a maioria dos programas de certificação requer usualmente o nível de mestrado, antes Que o candidato se submeta ao exame de certificação.

Para Manton ${ }^{(10)}$, certificação é o processo pelo Qual uma agência ou associação reconhece Que se alcançou certos padrões prédeterminados e definidos para a prática especializada daeuela profissão. Seu intuito é assegurar publicamente que o indivíduo se tornou expert naquele conhecimento e adquiriu habilidades em uma determinada especialidade. A certificação indica Que o profissional voluntariamente se engajou em um processo de autoevolução.

Nos EUA, a acreditação, diferentemente da prova para a licença da RN/LPN e da certificação da RN, evoluiu para avaliar instituições mais propriamente do Que indivíduos ${ }^{(9)}$.

Atualmente, naquele país, a Enfermagem é constantemente relembrada de sua responsabilidade de assegurar sua competência, pois, Quando um paciente entra em um hospital, espera Que o atendimento seja feito por um enfermeiro competente. Os pacientes e seus familiares não pensam necessariamente em competências definidas, mas eles consideram Que a Enfermagem é competente e Que o hospital adotou medidas para garantir Que o enfermeiro ofereça cuidado seguro, efetivo e apropriado ${ }^{(1)}$. Por isso, cada hospital deve, em particular, se assegurar da competência da equipe de enfermagem. Do enfermeiro é esperado Que ofereça segurança para o paciente e sua família, e isso se estende para as agências reguladoras, como é preconizado pela Joint Commission for Accreditation of Healthcare Organization (JCAHO) ${ }^{(1)}$.

Não há um consenso Quanto à definição de competência ou competência continuada. Na literatura científica, observa-se Que a maioria das definições reflete o contexto onde foram desenvolvidas. Os educadores entendem competência como uma mistura de conhecimento, prática, atitudes e julgamento. Atribuir competência não é uma tarefa fácil e não há uma resposta correta ou definição completa de competência ou avaliação de competência ${ }^{(1)}$.

Para outros autores, competência tem significados distintos, Que podem estar ligados a tarefas, resultados e características de pessoas. Segundo Fleury ${ }^{(12)}$, competência tem sido definida como "saber agir responsável e reconhecido Que implica mobilizar, integrar, transferir conhecimentos, recursos e habilidades Que agreguem valor econômico à organização e valor social ao indivíduo."

A noção de competência tem aparecido nos últimos anos como uma forma de repensar as interações entre as pessoas, seus saberes, suas capacidades e as organizações, suas demandas no campo dos processos de trabalho essenciais e os processos relacionais ${ }^{(13)}$. Sabe-se porém, Que a construção de um perfil de competência, por si só, não garante a renovação das práticas educativas em saúde. È necessário investir também na discussão e reflexão sobre a forma como esse perfil pode ser operacionalizado no cotidiano dos serviços e no ensino de enfermagem ${ }^{(14)}$.

Alguns dos métodos disponíveis para avaliação de competência são modelos de auto-avaliação ou provas com respostas de múltipla escolha, usados para certificação e licença, prova oral ou escrita e prática, ou com simulação de paciente ${ }^{(I I)}$.

Finalmente, é preciso lembrar Que a acreditação/certificação também tem a finalidade de atualização constante do enfermeiro para manter suas competências técnico-científicas a fim de serem mais valorizados pelos pacientes e mercado de trabalho, pois poderão comprovar seu aprimoramento constante, frente aos novos conhecimentos da área profissional.

\section{Acreditação dos Cursos de Graduação em Enfermagem}

Nos EUA, encontraram-se duas grandes entidades Que se dedicam a avaliar cursos e instituições de ensino de Enfermagem CCNE (Commission on Collegiate Nursing Education) e NLNAC (National League for Nursing Acrediting Commission) ${ }^{(15)}$.

Até 1998, o único organismo de acreditação profissional em Enfermagem era a National League for Nursing (NLN). Em 1996. a Commission on Collegiate Nursing Education (CCNE) foi estabelecida para a acreditação do bacharelado e do programa de pós-graduação em Enfermagem ${ }^{(15)}$.

A NLN foi fundada em 1952, Quando muitas organizações nacionais de Enfermagem emergiram nos EUA. A atividade de acreditação começou no início do século XX, Quando foi consolidada, na NLN, a Divisão de Educação em Enfermagem. Quatro conselhos a compuseram: Council of Association Degree Programs, Council of Baccalaureate and Higher Degree Programs, Concil of Diploma Programs e o Concil of Pratical Nursing 
Programs. Cada Conselho desenvolveu suas próprias políticas e procedimentos, e sua filosofia para a acreditação. Em 1995, para a aprovação governamental da NLN, foi recomendado Que os Quatro Conselhos adotassem um propósito comum para os padrões e critérios para a acreditação ${ }^{(15)}$.

Assim, em 1996, a National Advisory Committee on Institutional Quality and Integrity (NACIQI) do Departamento de Educação dos Estados Unidos recomendou Que a Secretaria de Educação não renovasse o reconhecimento da NLN como uma agência de acreditação. A recomendação foi sugerida por preocupações nas seguintes áreas: padrões obrigatórios de acreditação, inspeções não agendadas, monitoramento de instituições e programas, a necessidade de um programa de avaliação sistemática para estimar validade e credibilidade, equipe administrativa, finanças, treinamento de avaliadores do programa e controles para prevenir aplicações de padrões inconsistentes e decisões baseadas em critérios publicados. Após a indicação destas recomendações, feito pelo NLN, a Secretaria de Educação enviou o processo de volta ao $\mathrm{NACIQI}$ para maiores considerações, em seu encontro em junho de 1997. Isto resultou na fundação da National League for Nursing Acrediting Commission (NLNAC) em 1996, como um organismo independente, dentro da NLN, Que começou a funcionar em $1997^{(15)}$.

A Commission on Collegiate Nursing Education (CCNE) foi estabelecida em 1996 pela Associação Americana de Enfermeiros (American Nurses Association - ANA), como um organismo de acreditação autônomo para associados, o Qual é responsável pelo processo de acreditação e definição de políticas Que afetam a $\mathrm{CCNE}^{(13)}$. Deste então, vem promulgando "Position Statements" sobre o assunto, dos Quais, o último, de abril de 2008 é bastante abrangente ${ }^{(16)}$.

Em um tempo em Que os enfermeiros identificam a educação permanente como elemento essencial no cuidado com a saúde no ambiente de trabalho, o processo de acreditação pela CCNE oferece uma oportunidade singular de desenvolvimento profissional também para os enfermeiros oriundos da prática clinica, denominados "practicing nurses - PN", Que fizeram uma parceria com a CCNE voltada para a atividade de acreditação, colocando suas habilidades clinicas sob desafio ${ }^{(17)}$

A inclusão do primeiro grupo dos $\mathrm{PN}$ na equipe de avaliadores do CCNE se deu em outubro de 2000. Até 2005, o CCNE tinha $185 \mathrm{PN}$ que tinham participado da equipe dos avaliadores in loco e servido tanto como membros Quanto lideres das equipes de avaliadores, para mais de 377 programas de bacharelado em enfermagem e 267 programas de mestrado (localizados em 397 instituições nos EUA), Que foram acreditados pela $\mathrm{CCNE}^{(17)}$.

O sucesso desse processo, Que representa uma nova forma de colaboração entre a academia e os serviços, depende de um continuo fluxo dos PN Que têm treinado a avaliação in loco. Esses enfermeiros são o elo entre a educação acadêmica e a prática clínica; eles podem ser um modelo para os estudantes, demonstrando uma maneira na Qual a educação e os serviços de saúde se articulam, e também trazer experiência da prática clínica para os enfermeiros Que irão passar pelo processo de acreditação ${ }^{(17)}$.

Em resposta às mudanças no sistema de oferta do cuidado em saúde nos EUA, envolvendo o incremento de modelos assistenciais voltados para o gerenciamento dos sistemas de cuidado, o aumento do uso dos serviços pelos pacientes externos e o aumento do cuidado oferecido pelas bases comunitárias, o bacharelado em enfermagem está mudando. A reorientação se dirige para incorporar a experiência do aprendizado do estudante em situações de base comunitária, o Que também influencia na avaliação. Assim, a acreditação se tornou um mecanismo para avaliar a Qualidade desses programas educacionais, sendo Que a acreditação pela CCNE oferece o processo para o acesso a programas de Qualidade para enfermeiros, eneuanto encoraja a flexibilidade e inovação nesses programas $^{(18)}$ de ensino.

Como se viu, nos EUA há mais de um órgão Que se dedica a acreditação, o Que indica Que esses processos são valorizados pelos profissionais e órgãos de controle de serviços de saúde.

\section{Acreditação dos Programas de Educação Continuada para Enfermeiros}

Em 1899, foi planejado e executado um dos primeiros programas de Educação Continuada formalizados nos EUA: um curso de economia hospitalar, na Columbia University. Depois disso, as associações de escolas de enfermagem e hospitais passaram a oferecer cursos especializados para seus membros ${ }^{(4)}$.

A partir de 1920, a Educação Continuada para enfermeiros começou a ser disponibilizada através de instituições e conferências, oferecendo instruções em áreas como: doenças infecciosas, centro cirúrgico, enfermagem psiQuiátrica e enfermagem em tuberculose. As mudanças no ambiente de trabalho em saúde nos anos 1960 , decorrente de pressões públicas, intervenção governamental e o consentimento profissional, transformaram um sistema relativamente simples, criado para facilitar a atualização profissional, em um complexo mecanismo destinado a assegurar a competência dos profissionais, aumentando o interesse na acreditação em Educação ${ }^{(4)}$.

O desenvolvimento de padrões para a Educação Continuada em Enfermagem, no entanto, surgiu apenas após a instituição de um Conselho de Educação Continuada. Isso ocorreu em 1974, Quando a Casa de Delegados da ANA aprovou uma resolução histórica, estabelecendo um sistema de acreditação para Programas de Enfermagem de Educação Contínua ${ }^{(4,17)}$ e, em 1975, um comitê de estudos independente iniciou o processo. Como primeira medida, a Comissão de Educação Continuada da ANA delegou, ao Conselho de Educação Continuada (atualmente o Conselho Profissional de Enfermagem para Educação e Desenvolvimento), a responsabilidade de desenvolver os critérios de acreditação. Quase Que concomitantemente, respondendo ao crescimento e antecipando as contínuas necessidades da Qualidade da educação continuada, um novo modelo de acreditação foi voluntariamente implementado em provedores estatais da Educação Continuada em Enfermagem, regionalmente, estendendo-se para o nível nacional, no final dos anos $1970^{(4)}$.

A primeira acreditação formalmente concedida ocorreu em 1976. No início, a National Accreditation Board (NAB) administrava o sistema de acreditação da Associação Americana de Enfermagem (ANA) através de cinco Comitês Regionais e um Comitê Nacional. Eles avaliavam e acreditavam colégios e universidades, programas de preparação para enfermeiros Que não eram de graduação, Quadros estatais de enfermagem, associações estaduais de enfermagem, organizações nacionais de especialistas e serviços 
de enfermagem federais ${ }^{(4,20)}$.

Depois Que a American Nurses Credentialing Center (ANCC) foi incorporada, em janeiro de 1991, como subsidiária da ANA, a Commission on Accreditation (COA) foi estabelecida como Órgão de Acreditação. Essa comissão é composta por 14 membros eleitos, Que operam o programa de acreditação, desenvolvem a política e mantêm o alinhamento do programa com a prática educacional. Os membros são representantes de vários órgãos como universidades e associações. Um dos membros da COA é uma enfermeira - RN, Que tem a maior responsabilidade das atividades de educação continuada na organização provedora de acreditação. Outro membro é a RN representante dos Que provêem o cuidado direto ao paciente. Ainda há aquela com título de doutor, expert em avaliação e pesQuisa e outro um membro Que não é enfermeiro, mas com doutorado, docente de universidade, com especialização em Educação de Adultos ou Educação Continuada(18). Em 2000, 273 organizações procuraram e obtiveram acreditação através da ANCC.

A acreditação em Educação Continuada em Enfermagem nos EUA ocorre em três níveis: nacional, organizações especializadas e pelos Estados da federação. No nível nacional, organizações como universidades, associações estaduais de enfermagem, empresas de produtos comerciais e serviços de enfermagem federais podem se tornar provedores acreditados de Educação Continuada. A maioria dessas organizações é submetida a um exame formal para tornaremse acreditadas nacionalmente. Organizações Qualificadas podem escolher serem fornecedoras da Educação Continuada em Enfermagem através de associações estaduais de enfermagem e/ ou organizações especializadas em enfermagem ${ }^{(4)}$.

Ainda naquele país, em fevereiro de 2006, uma força tarefa de sete experts no campo da Educação Continuada, representando universidades e associações estaduais de enfermeiros, começou a escrever Questões de excelência, baseada na revisão de literatura e em programas de Qualidade excepcional. Foram identificados 11 programas nessa condição, atribuída a fatores como criatividade, inovação, liderança em áreas de educação, pratica de negócios, expansão e disseminação de conhecimento ${ }^{(21)}$.

Apesar dos maiores responsáveis pela educação continuada serem os próprios profissionais de enfermagem, seus empregadores também podem oferecer suporte através de uma estrutura de educação continuada Que têm a intenção de assegurar educação de alta Qualidade e em beneficio do consumidor do cuidado de enfermagem $^{(20)}$. Por isso, a maioria das organizações Que oferecem cuidados com a saúde participa de algum tipo de acreditação em Educação Continuada ${ }^{(4)}$, estendendo-se essa ação para os enfermeiros Que atuam em áreas especializadas. Nesse contexto, assim como ocorreu na Educação Continuada, o Comitê Nacional da Emergency Nurses Association (ENA) e o seu Departamento de Educação estão propiciando a obtenção dos créditos das horas computadas nos programas de educação continuada, para os enfermeiros nos EUA ${ }^{(22)}$.

Interessante notar Que as áreas de especialização já têm mecanismos próprios, o Que prova Que, na cultura americana, a idéia da acreditação já esta bem desenvolvida.

\section{Certificação e Recertificação de Enfermeiros Especialistas}

Nos EUA, os enfermeiros generalistas são avaliados principalmente pelo NCLEX (National Council's Learning Extension) e os especialistas por entidades de classe específicas ou por empresas que a estas prestam serviços. Para prestar exame, cada modelo de certificação faz várias exigências, como anos de experiência na prática clinica e experiência em procedimentos específicos $^{(23)}$.

O tempo pelo Qual o enfermeiro é certificado varia entre as especialidades da Enfermagem. Devido à crescente tendência de Questionar se a Educação Continuada fornece uma medida de competência, vários outros métodos estão sendo desenvolvidos para a recertificação.

A necessidade de adotar critérios adequados para a certificação no cuidado em emergência levou a ENA a desenvolver um currículo padrão e uma prova de certificação para enfermeiros de emergência. A oportunidade de se apropriar de mais conhecimento aumenta a competência e a Qualidade da prestação de serviço em saúde. Nessa perspectiva, o processo de certificação funciona como fator motivacional em direção a excelência, como uma recompensa e como garantia para o público (consumidor de serviços de saúde) e para os administradores das instituições de saúde ${ }^{(24)}$.

Há muito interesse da Inglaterra e dos Estados Unidos na educação para a prática avançada da enfermagem (Advanced Pratice Nurses - APNs), principalmente Quanto aos seus procedimentos, especialmente aqueles relacionados ao cuidado de emergência. Existem Quatro categorias de Pratical Nurses (PN) nos EUA: enfermeira pratica (nurse practitioner - NP), enfermeira clínica especializada (clinical nurse specialist - CNS), enfermeira anestesista registrada e certificada (certified nurse anestesist- CRNA) e enfermeira parteira certificada (certified nurse midwife - CNM). Nos EUA, as APNs mais comumente encontradas no setor de cuidados de emergência são NPs e CNSs ${ }^{(25)}$.

O critério para renovação da certificação varia de Estado para Estado e pode incluir o acúmulo específico de número de horas de prática clínica e créditos em Educação Continuada, desde o período de renovação. Dois exemplos de agências certificadoras são: American Nurses Credentialing Center (ANCC) e a American Academy of Nurse Practitioners (AANP). A ANCC também requer a complementação do número de horas clínicas específicas no programa da APN - 500 horas para a CNS, por exemplo, como também um curso de farmacologia avançada, avaliação da saúde e patofisiologia. Uma vez certificada, a APN tem Que passar por uma nova medida de Qualificação para a recertificação ${ }^{(25)}$.

A Emergency Nurse Association (ENA) tem atuado no nível nacional, desenvolvendo padrões para a prática para NPs e CNs, no cuidado de emergência. O campo de atuação das NPs no cuidado de emergência requer Que elas estejam preparadas no nível de mestrado, para proporcionar alta Qualidade do cuidado ${ }^{(25)}$.

Com a freqüente ênfase no credenciamento de Enfermagem, muitos enfermeiros têm procurado fazer o exame para a Certified Emergency Nurses (CEN). A ENA desenvolveu material educacional Que pode ser usado como outro recurso para o preparo para obtenção do CEN, composto pelo Emergency Nursing Core Curriculum e o CEN Review Manual (26).

Para os enfermeiros da área cirúrgica, a Certificaton Board Perioperative Nursing (CBPN), nos EUA, reconhece Que a aprendizagem e a manutenção da competência são realizadas de várias maneiras, seja pelo aprendizado obtido através das atividades 
profissionais, ou pela conclusão do portfólio profissional pré e pósoperatórios; dessa maneira eles vão se certificando e/ou renovando a certificação, nos prazos estabelecidos. O Comitê de Pesquisa CBPN e a Nurse Credentialing Research Coalition (NCRC) são comitês para Quantificar não somente o valor da certificação, mas para examinar os resultados pessoais, profissionais e práticos de enfermeiros certificados no país ${ }^{(1)}$.

A recertificação é um componente necessário para o processo de certificação e pode ser realizada de muitas maneiras. O CBPN, por exemplo, realiza um exame para o enfermeiro que trabalha no pré e pós operatório, ou submeter à avaliação, as horas computadas para crédito em educação continuada e, mais recentemente, instituíu a recertificação por pontos obtidos por meio de freqüência de cursos $^{(1)}$.

Aos certificantes é permitido usar pontos computados por meio da freeüência a palestras, ou autoria de publicações ou regência de cursos. As publicações de artigos ou capitulo de livro, apresentação de palestra de 30 a 60 minutos ou seminários de 4 a 8 horas, incluindo o Congresso da Association of periOperative Registered Nurse (AORN), Que também atribui pontos, podem ser usados para recertificação. Outra forma de acumulação de pontos é o treinamento numa nova área de enfermagem transoperatória tais como: enfermagem endoscópica, obstétrica, anestesia, ou cateterismo cardíaco ${ }^{(11)}$.

lá, na Espanha, os processos de acreditação e certificação começaram no final de 1990. Os cuidados complexos e sofisticados, Que só podem ser proporcionados ao paciente por uma equipe de profissionais altamente Qualificados e paralelamente a um grande desenvolvimento tecnológico, suscitou a preocupação dos profissionais de cuidados intensivos em Qualificar os recursos humanos. Todas essas mudanças, somadas à tendência dos centros hospitalares em alcançar altos padrões de Qualidade na atenção ao paciente, tornaram imprescindível Que os enfermeiros alcançassem alto nível de competência profissional, o Que incluía possuir conhecimentos, habilidades, valores e atitudes para o desenvolvimento de sua atividade ${ }^{(23)}$.

Existem poucas diretrizes internacionais Que descrevem as competências que requerem os enfermeiros de cuidados intensivos. Alguns países como Canadá, África do Sul e Austrália estabeleceram padrões nacionais de conhecimento, necessários para a atenção do paciente crítico. Para alcançar a competência profissional, o enfermeiro necessita de desenvolvimento profissional contínuo. Por esta razão, é adequado realizar avaliações periódicas, para identificar se os profissionais alcançaram o nível de competência exigido e dispõem do preparo requerido para desenvolver sua atividade $^{(23)}$

Na Espanha, a Sociedade Espanhola de Enfermagem Intensiva e Unidades Coronarianas (SEEIUC) oferece a possibilidade de obter um diploma Que acredita a competência profissional no cuidado ao paciente crítico. A obtenção da certificação exige cumprimento dos requisitos estabelecidos para a prática assistencial ativa e aprovação em prova escrita de conhecimentos. O programa de certificação estabelece recertificações a cada três anos, mediante o reconhecimento de pontos de educação continuada (obtidos através de publicações ou créditos de educação continuada, dentre outros) e com um novo exame, sendo Que, em QualQuer caso, se exige a prática clinica do cuidado ao paciente crítico $^{(23)}$.

Nos últimos 40 anos, no julgamento de um estudioso do assunto $^{(27)}$, a formação de profissionais de saúde tem tido significativa melhora em direção a assegurar o preparo profissional através da certificação individual e de programa aprovado e acreditado. No Brasil, algumas sociedades de especialistas têm desencadeado esta ação de desenvolvimento e regulação profissional, porém de maneira incipiente ${ }^{(6,28)}$. Uma solução em potencial poderia ser a proposição de programas para a certificação de enfermeiros especialistas. Esta demonstraria que o enfermeiro alcançou um nível específico de conhecimento em uma determinada área da enfermagem e atestaria um conjunto determinado de competências por algum tempo, Quando nova avaliação deveria ser empreendida.

\section{CONCLUSÃO}

A história mostra Que os países Que têm tecnologia mais desenvolvida no cuidado à saúde, como os EUA e a Espanha, por exemplo, já praticam a certificação e acreditação há algum tempo.

A acreditação nasceu vinculada à Educação Continuada, Que era praticada de forma livre, sem essa conotação, mas rapidamente evoluiu para ser um mecanismo de controle de Qualidade do profissional.

A multiplicidade de organismos Que se ocupam da acreditação e certificação são uma medida da valorização dessa prática, nos locais onde elas estão instituídas. Os critérios são variados, a forma de operacionalização também, e ambos estão vinculados à especificidade da área em Que são acreditados ou certificados.

A cultura de avaliação para garantir a Qualidade em saúde no lugar em Que se a pratica, trabalha com as três dimensões: a Escola, os Serviços e os Profissionais.

Chamou à atenção também Que as escolas são acreditadas e não só o profissional e Que, em certa medida, a Educação Continuada é o agregador das três dimensões de avaliação porQue faz a ponte entre o serviço e a escola, através do profissional.

Dessa forma, pode-se concluir Que os processos de certificação e acreditação profissional para enfermeiros e escolas são praticados principalmente em países desenvolvidos, iniciou-se no meio do século passado e merecem ser estudados com mais profundidade, principalmente os dos enfermeiros especialistas, sobre o Quê se encontrou pouca informação.

\section{REFERÊNCIAS}

I. Caverni L. Qualidade da assistência e acreditação profissional. Rev Paul Enferm 2005; 24 (4): 1-3

2. Consórcio Brasileiro de Acreditação (CBA). O Que é acreditação? [citado em 2 I set 2008]. Disponível em: http:// www.cbacred.org.br

3. Associação Brasileira de Normas Técnicas (ABNT). Certificação. [citado em 21 set 2008]. Disponível em: http:/ /www.abnt.org.br

4. DeSilets, LD. Accreditation of Continuing Education: the critical elements. I Contin Educ Nurs 1998; 29(5); 204-38. 
5. Holleran, RS. Air \& Surface patient transport- Principles \& Pratica. Saint Louis: Mosby; 2003.

6. Conselho Federal de Medicina (CFM) - Resolução CFM $n^{\circ}$ 1.772/2005 - Dispõe sobre a Comissão Nacional de Acreditação - Certificação de Atualização Profissional. Diário Oficial da União 2005 ago 12; I: 14 I-2.

7. Takahashi RT, Oguisso T. Reflexões sobre a certificação profissional de enfermagem no Brasil. Rev Paul Enferm 2005; 24(4): 37-42.

8. Biblioteca Virtual em Saúde - Literatura Científico-Técnica [citado em 14 jun 2008]. Disponível em: http:// www.bibliotecavirtualemsaude.org

9. Barnum BS. Licensure, Certification, and Accreditation. I Issues Nurs [serial online] 1997 aug 13. [cited 2008 jun 14]. Available at: http:/www.nursingworld.org/ojin

10. Manton A. Reneeal of certification by examination- why? I Emerg Nurs I 984; 10 (3): 30A-3 I A.

1 I. O'Neale M, Kurtz S. Certification:perspectives on competency assurance. Semin Perioper Nurs 200 I; 10(2): 88-92.

12. Cunha ICKO, Ximenes Neto FRG. Competências gerenciais de enfermeiras: um novo velho desafio? Texto Contexto Enferm 2006; 15(3).

13. Ruthes RM, Cunha ICKO. Entendendo as competências para aplicação na enfermagem. Rev Bras Enferm 2007; 6I(1): 109-12.

14. Leonello VM, Oliveira MAC. Competências para ação educativa da enfermeira. Rev Latino-am Enfermagem 2008; $16(2)$.

15. Overbay ID, Aaltonem PM. A comparison of NLNAC and CCNE accreditation. Nurse Educ 200 I; 26(1): 17-22.

16. American Association of Colleges of Nursing. The Essentials of Baccalaureate Education for Professional Nursing. Practice [cited 2008 may 27]. Available: http://www.aacn.nche.edu/
Publications/positions/scholar.htm

17. Beason CF. A new role in acreditation activities offers expanded horizons for registered nurses. I Profes Nurs 2005; 2 I (3): 191-6.

18. Ort SV, Townsend I. Community-based nursing education and nursing accreditation by the Commission on Collegiate Nursing Education. I Profes Nurs 2000; 1 I6(6): 330-5.

19. Bell DF, Matthews I. Quality standards for acreditation of continuing education. JONA 1998; 28(12): 15-6.

20. Ianvier, KA. Pursuing ANA accreditation as a provider of continuing education in nursing. I Nurs Staff Develop 1990; 6(6): $275-8$

21. DeSilets LD. Taking accreditation to the next step. J Contin Educ Nurs 2006; 37(6): 245.

22. Walsh R. Obtaining CECH approval through ENA: the process has been made easier. I Emerg Nurs 200 I; 27(6): 594-6.

23. Erro, M.C.A. La competência profesional y la acreditacion de enfermeras em el cuidado del paciente crítico. Enferm Intensiva 2005; 16(1): 1-2.

24. EDNA report. J Emerg Nurs 1975; I (2): 32-4.

25. Cole FL. Emergency care. Advanced pratice nursing in the US: an overview. Emerg Nurse 2003; 1 I (5): 22-5.

26. Burlington KY. Knowledge assessment and preparation for the certified emergency nurses examination. I Emerg Nurs 2007 33(2): 138-9.

27. Allegrant JP, Airhihenbuwa CO, Auld ME, Birch DA, Roe KM, Smith BJ. Nacional Task Force on accreditation in health education. Health Educ Behav 2004; 3 I (6): 668-83.

28. Sociedade Brasileira de Enfermagem em Centro Cirúrgico e Centro de Material. Revalidação do título de especialista. [citado em 14 jun 2008]. Disponível em: http:// www.sobecc.org.br. 